\title{
Moving kinks and nanopterons in the nonlinear Klein-Gordon lattice
}

\author{
A. V. Savin ${ }^{a}$, Y. Zolotaryuk ${ }^{b, 1,2}$ and J. C. Eilbeck ${ }^{\text {b }}$ \\ ${ }^{\mathrm{a}}$ Institute for Problems of Physics and Technology, Moscow 119034, The Russian \\ Federation \\ ${ }^{\mathrm{b}}$ Department of Mathematics, Heriot-Watt University, Edinburgh EH14 4AS, UK
}

\begin{abstract}
We study moving topological solitons (kinks and antikinks) in the nonlinear KleinGordon chain. These solitons are shown to exist with both monotonic (non-oscillating) and oscillating asymptotics (tails). Using the pseudo-spectral method, the (anti)kink solutions with oscillating background (so-called nanopterons) are found as travelling waves of permanent profile propagating with constant velocity. Each of these solutions may be considered as a bound state of an (anti)kink with a background nonlinear periodic wave, so that the wave "pushes" the (anti)kink over the Peierls-Nabarro barrier. The stability of these bound states is confirmed numerically. Travelling-wave solutions of permanent profile are shown to exist depending on the convexity of the on-site (substrate) potential. The set of velocities at which the (anti)kinks with monotonic asymptotics propagate freely is calculated. We also find moving nonoscillating (anti)kink profiles with higher topological charges, each of which appears to be the bound state of (anti)kinks with lower topological charge $(|Q|=1)$.
\end{abstract}

Key words: Nonlinear lattice; Topological solitons; Nanopterons; Pseudo-spectral method

Pacs numbers: 03.40.Kf, 63.20.Ry.

\footnotetext{
1 Current address: Max-Planck-Institut für Physik komplexer Systeme, Nöthnitzer Str. 38, 01187 Dresden, Germany

2 Corresponding author. Tel.: +49-351-871-1203; fax: +49-351-871-1199; e-mail: yzolo@mpipks-dresden.mpg.de.
} 


\section{Introduction}

It is known [1] that in some limiting cases, a chain of interacting particles, subject to a nonlinear on-site (substrate) potential with two or more degenerate minima, supports moving topological solitons as monotonically increasing (kink) or decreasing (antikink) travelling-wave solutions. By travelling waves, we mean waves of stationary profile moving with constant velocity. (In particular, we will frequently consider the case where a kink moves on a background of a small amplitude oscillatory wave of infinite extent, travelling at the same velocity as the kink).

In general, while propagating along the chain, the kink radiates small-amplitude waves and finally stops because of the existence of the so-called Peierls-Nabarro $(\mathrm{PN})$ potential barrier (see, e.g., ref. [1]). However, the topological soliton (kink and antikink) solutions appear to be well-defined travelling waves of stationary profile while they are moving on an appropriate oscillating background [2]. If the inter-particle potential is anharmonic, the soliton solution obtained in ref. [3] describes the antikink moving without radiation when its velocity attains certain fixed values. This solution has the properties of both topological and acoustic (lattice) solitons and its existence is due to some balance of on-site and inter-particle nonlinearities. In the case of harmonic inter-particle interaction, such "transparent" motion can also exist, but only for topological charge $|Q|>1$ and also with some fixed velocities (see refs. [1,3]).

On the other hand, the discretisation of the continuous sine-Gordon (SG) equation used in refs. [4,5] yields a discrete integrable system which admits monotonic topological soliton solutions. However, such a discrete version does not describe a standard system of coupled particles, particularly, the KleinGordon chain. Another discretisation procedure has been used in ref. [6] for the SG equation and in ref. [7] for the $\phi^{4}$ model. For both systems, the PeierlsNabarro $(\mathrm{PN})$ potential barrier was eliminated and, as a result, a discrete system of equations have been obtained, describing transparent regimes of kink propagation.

As is commonly known (see, e.g., ref. [8]), in the continuum limit the nonlinear Klein-Gordon equation admits moving kink solutions with a finite interval of velocities from zero to the speed of small-amplitude waves. These kink solutions have monotonic behaviour of the standard type. The interaction of a kink with small-amplitude waves (phonons) was a topic of great interest for two decades and the details of these investigations are given in the review papers by Bishop et al. [8] and Jackiw [9]. In particular, it was shown that a small-amplitude wave can propagate transparently through a standing kink with some change in the wave phase which depends on the velocity of wave propagation. In this case the wave is not trapped by the kink and therefore 
such a solution does not belong to the class of pure travelling waves. On the other hand, the bound states of a kink and a wave moving with the same velocity were studied in a generalised version of the continuous SG equation with nonlocal interactions [10]. These solutions can be referred to as kinks on an oscillating background or as nanopterons, the term introduced by Boyd in the case of continuous systems [11] (see also the recent review by Flach and Willis [12]).

The present paper aims at studying topological soliton solutions that have both non-oscillating (monotonic) and oscillating tails. Note that in the latter case (nanopterons), the tails propagate with the same velocity as the kink itself. This new type of solution appears to be stable, resulting in the formation of a bound ("self-trapped") state of a kink and a wave with a nonlinear dispersion law. As a result of this interaction, the phase velocity of phonons decreases to sub-sonic values. On the other hand, due to the kink-wave interaction, the kink is able to propagate in a discrete chain with any constant subsonic velocity, despite the existence of the PN barrier. In other words, the kink is pinned to the wave decreasing its velocity and in its turn this wave forces the kink to propagate in a coupled way, i.e., some kind of the dynamical self-trapping mechanism of the kink with the (nonlinear) wave takes place.

We also study the structure of the nonlinear waves themselves. Similarly to the continuous Klein-Gordon system that supports nonlinear periodic waves (so-called cnoidal waves), we expect that nonlinear waves of the same kind can also exist in the discrete case. However, these waves should possess specific features of discrete systems.

The following important result concerning kink mobility, has been obtained in refs. $[13,14]$. Here the model with the on-site periodic potential constructed from pieces of parabolas has been considered. In this model the switching of the ground state from the kink solution centered on a chain site to the kink solution, centered in-between two sites and vice-versa depending on the onsite potential convexity was shown to occur. This observation gives a good reason to believe that for a general nonlinear on-site potential, depending on its convexity, a transparent regime of the kink propagation can occur as well.

In the majority of cases, due to symmetry arguments, the PN barrier that describes the pinning potential experienced by narrow kinks is defined through the modulus of the energy difference $\Delta E=\left|E_{0}-E_{1 / 2}\right|$ where $E_{0}\left(E_{1 / 2}\right)$ is the energy of a kink centred on a chain site (in-between two sites), as described in [15]. However, as shown by Peyrard and Remoissenet [16], strictly speaking, this definition is not correct in all cases because the two particular states 0 and $1 / 2$ do not always have highest and lowest potential energies. Therefore in a general case, the height of the PN barrier should be calculated taking into account all intermediate positions of a kink (or an antikink) within an 
unit chain cell [17]. On the other hand, it should be noticed that in itself the absence of a PN barrier is not a condition for the existence of a travelling wave solution of constant profile because the PN barrier is defined for static (standing) kinks, i.e., when the kink velocity $s=0$.

If for a certain value of the kink velocity $s \neq 0$, there exists a moving kink of permanent profile, this happens for some parameter values of the chain that in general differ from those for which the switching of the kink ground state occurs. Consequently, if we find a set of the chain parameter values for which $\Delta E=0$, one can expect that for a slightly changed set of parameters there exists a moving kink solution with some small, but non-zero value of the velocity $s$. In the present paper, we find these parameter values, starting from an infinitesimal value of the velocity $s$, with subsequent continuation step by step for all admissible velocities.

It should be also mentioned that the existence of non-oscillating kink solutions with higher topological charges $(|Q|>1)$ has been demonstrated at some special values of the velocity. However, the mechanism of such a transparent regime differs from that discussed above. Thus, Peyrard and Kruskal [1] explained this phenomenon as the result of the "resonance" interaction of the waves emitted by two kinks with topological charge $Q=1$ when these waves happen to be out-of-phase. The waves suppress each other, so that they annihilate and, as a result, the composite kink (with charge $Q=2$ ) propagates without radiation. The same situation can happen for $|Q|=3,4, \ldots$. Such non-oscillating topological solitons can be considered as bound states of solitons (kinks or antikinks) with lower topological charge, formed due to attractive interaction through phonons. We expect that more sophisticated configurations (profiles) of such bound states which may be similar to those obtained in ref. [10], can exist in the discrete Klein-Gordon lattice. It is also worth of mentioning results on damped and d.c. driven Josephson-junction arrays where similar kink (fluxon) structures have been studied (see, e.g., refs. $[18,19])$.

The paper is organised as follows. In Section 2 we describe the model and derive the equations of motion. The linear and nonlinear periodic wave solutions to our model are studied in the next section. In Section 4 we investigate the Peierls-Nabarro barrier for standing kink solutions. Section 5 is devoted to moving kinks: both with monotonic and oscillating tails. Concluding remarks and a discussion are contained in Section 6 . 


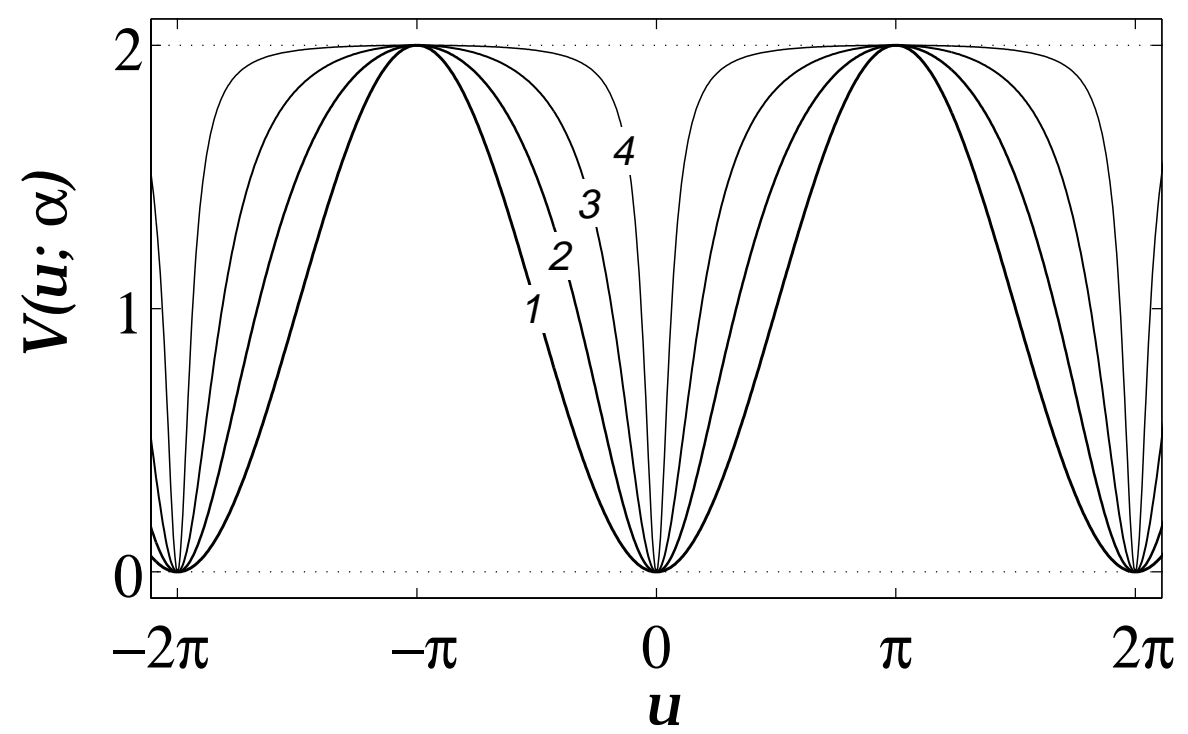

Fig. 1. The shape of the on-site potential $V(u ; \alpha)$ for $\alpha=0$ (curve 1), $\alpha=1$ (curve 2 ), $\alpha=5$ (curve 3 ), $\alpha=20$ (curve 4 ) and $\gamma=1$.

\section{The model}

We consider a chain of coupled particles subjected to an external on-site potential. The dimensionless Hamiltonian of such a system can be written as follows

$$
H=\sum_{n \in \mathbb{Z}}\left[\frac{1}{2} p_{n}^{2}+U\left(u_{n+1}-u_{n}\right)+V\left(u_{n}\right)\right]
$$

where $p_{n}=\dot{u}_{n}$ is the conjugate momentum of the $n$th particle in the chain, $u_{n}$ is the displacement of the $n$th particle from its equilibrium position, and $U(r)$ is the inter-particle interaction potential normalised by $U(0)=0$ and $U^{\prime \prime}(0)=1$. We consider only a harmonic approximation to the inter-particle potential: $U(r)=r^{2} / 2$. The on-site potential $V(u)$ is assumed to be a symmetric periodic function with period $2 \pi$. Its minima are situated at $u=2 \pi k, \quad k \in \mathbb{Z}$, and maxima at $u=(2 k+1) \pi, \quad k \in \mathbb{Z}$. For the periodic function $V(u)$, we choose the slightly modified Peyrard-Remoissenet potential [16]:

$$
V=V(u ; \alpha)=\gamma \frac{(1+2 \alpha)(1-\cos u)}{1+\alpha(1-\cos u)}, \quad \gamma>0
$$

where the parameter $\alpha \geq 0$ measures the convexity of the on-site potential barrier and $2 \gamma$ is its height. The shape of the potential (2) for $\alpha=0,1,5$ and 20 is shown in Fig. 1. 
When $\alpha=0$, the function (2) coincides with the on-site potential for the SG model. For all values of the parameter $\alpha \geq 0$ the minima of this potential are 0 and the maxima are $2 \gamma$. The increase of $\alpha$ leads to the monotonic growth of the convexity of the barrier (see Fig. 1). In the limit $\alpha \rightarrow \infty$ the potential $V(u)$ tends to the discrete periodic potential

$$
V(u ; \infty)=2 \gamma \begin{cases}0, & u=2 \pi k, \quad k \in \mathbb{Z} \\ 1, & u \neq 2 \pi k, \quad k \in \mathbb{Z}\end{cases}
$$

The Hamiltonian (1) gives the discrete nonlinear Klein-Gordon equation:

$$
\ddot{u}_{n}=u_{n+1}-2 u_{n}+u_{n-1}-V^{\prime}\left(u_{n}\right), \quad n \in \mathbb{Z} .
$$

In this paper we focus on travelling-wave solutions of stationary profile moving with constant velocity $s$. For this type of solutions one can write

$$
u_{n}(t)=u(n-s t) \equiv u(z), \quad z=n-s t .
$$

Then eq. (4) is reduced to the following differential-difference equation:

$$
s^{2} u^{\prime \prime}(z)=u(z+1)-2 u(z)+u(z-1)-V^{\prime}[u(z)] .
$$

It is extremely difficult to perform a complete analysis of eq. (6) analytically and therefore, in order to find travelling-wave solutions in the form of periodic waves or (anti)kinks, we shall study this equation numerically using the socalled pseudo-spectral method [20].

\section{Periodic waves of stationary profile}

First, let us study the dispersion law for a nonlinear periodic wave. This wave can be considered as a linear wave only in the following two opposite limiting cases: (i) for small amplitudes $\left(\left|u_{n}\right|<<\pi\right)$ and (ii) for large amplitudes $\left(\left|u_{n}\right|>>\pi\right)$. In the small-amplitude limit the dispersion law can be written as follows

$$
\omega^{2}(q)=2(1-\cos q)+\gamma
$$

where $q=2 \pi / \lambda$ is the wave number and $\lambda \geq 2$ is the wave length. In the large-amplitude limit the on-site potential $V(u)$ essentially does not influence 
the wave propagation and therefore the dispersion law becomes approximately

$$
\omega^{2}(q)=2(1-\cos q) .
$$

The phase velocity of a linear wave depends only on its length: $s=\omega(q) / q$ (the velocity of small-amplitude long-length waves is $s_{0}=\lim _{q \rightarrow 0} \omega(q) / q=1$ ). Since the system of equations of motion (4) is nonlinear, the velocity of a periodic wave depends not only on its length, but also on its amplitude. We look for periodic travelling-wave solutions of the form (5) where $u(z)$ should be an even periodic function with period $L=\lambda$. Then this solution should have zero average:

$$
\int_{0}^{L} u(z) d z=0 .
$$

We can find such a solution by using the pseudo-spectral method which is briefly described as follows. Let the period $L$ and the velocity $s$ be fixed. Then we can look for the wave profile as the expansion in a finite Fourier series

$$
u(z) \simeq \sum_{j=1}^{N} c_{j} \phi_{j}(z)
$$

where $\phi_{j}(z)=\cos (2 \pi j z / L)$. The number $N$ of the coefficients $c_{j}$ should be chosen in such a way that the further increase of $N$ does not cause a change of the wave profile to a given numerical tolerance (usually, it is sufficient to choose $N=100$ ). Substituting the expansion (10) into eq. (6), for any $z$ we obtain the following expression:

$$
\begin{aligned}
F(z) & =\sum_{j=1}^{N}\left[\phi_{j}(z+1)-2 \phi_{j}(z)+\phi_{j}(z-1)+s^{2}\left(\frac{2 \pi j}{L}\right) \phi_{j}(z)\right] c_{j}- \\
& -V^{\prime}\left(\sum_{j=1}^{N} c_{j} \phi_{j}(z)\right)=0 .
\end{aligned}
$$

Next, calculating the function (11) for the $N$ collocation points $z_{i}=(i-$ 1) $L / 2(N-1)$, we obtain a system of $N$ algebraic equations with respect to the $N$ unknown coefficients $c_{i}$. To solve this system we apply the Powell hybrid method. We chose a set of coefficients $c_{1}=\pi, c_{2}=0, \ldots, c_{N}=0$ as an initial point for the iteration. Having found the coefficients $c_{i}$ 's, according to the series (10), one can reconstruct the (anti)kink shape.

The results of the numerical solution of eq. (6) have shown that, for any given value of velocity $s$, there exists a periodic wave of some stationary profile. The 
dependence of the wave amplitude $A=\sum_{j=1}^{N} c_{j}$ on its length $\lambda$ is given in Fig. 2 .

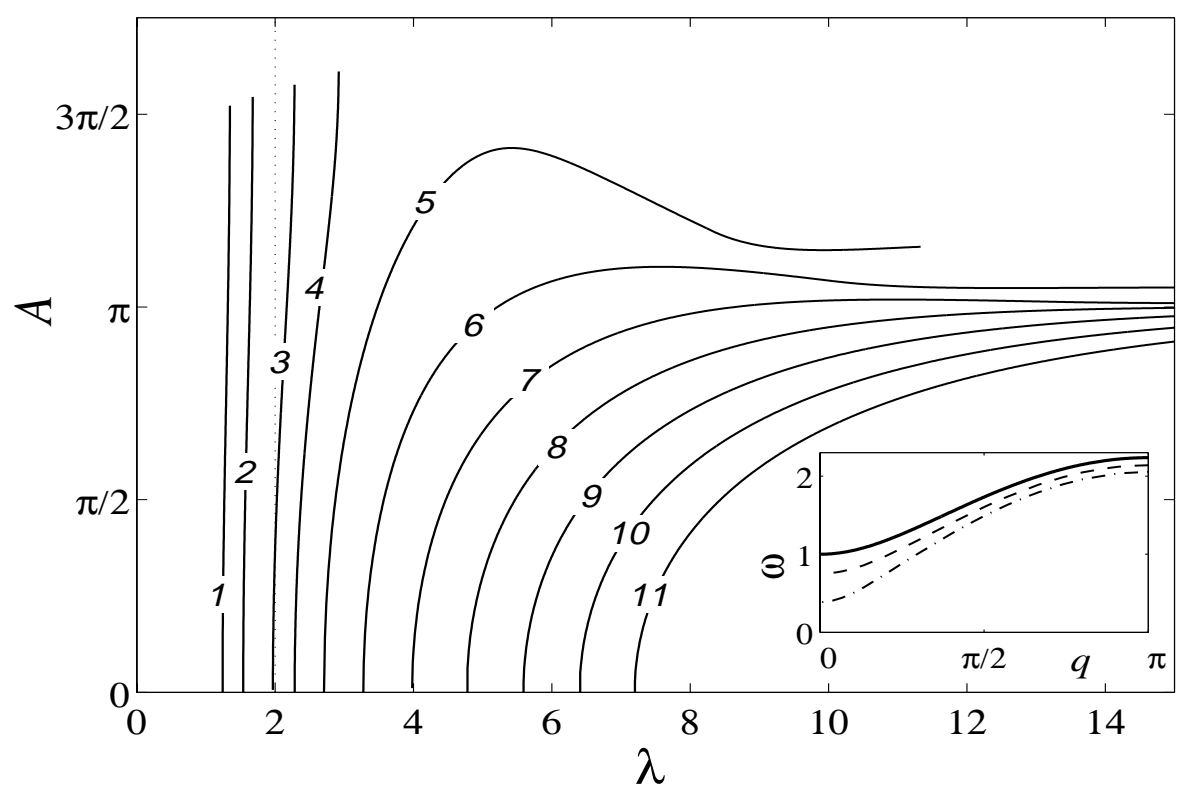

Fig. 2. Amplitude of the nonlinear periodic wave $A$ against its length $\lambda$ for $\alpha=0$, $\gamma=1$ and $s=0.3,0.5,0.7,0.8,0.9,1.0,1.1,1.2,1.3,1.4$ and 1.5 (curves 1 to 11, respectively). The inset shows the linear (solid line) and nonlinear dispersion laws (dashed line corresponds to the amplitude $A=2.0$ and dashed-dotted line corresponds to the amplitude $A=3.0)$.

It is interesting to note that in the region of $A>\pi$ there exists an region of points where there are no solutions, whereas for any pair $(A, \lambda)$ with $A \leq \pi$ there always exists a nonlinear periodic wave with these values for amplitude and length. The profile of these waves almost coincides with the shape of the sine function, except for waves with amplitude close to $\pi$ and length exceeding 10 (see Fig. 3).

\section{Pinning of kinks to the chain}

In this section we study static kink profiles and their corresponding PN potential barrier. In order to find these profiles, it is convenient to transform the Hamiltonian (1) to the form which explicitly contains the inter-particle coupling constant $\kappa=\gamma^{-1}$. As a result, the total potential $\left(p_{n} \equiv 0\right)$ chain energy [see eqs. (1) and (2)] becomes

$$
E=\sum_{n \in \mathbb{Z}} E_{n} \equiv \sum_{n \in \mathbb{Z}}\left[\frac{1}{2} \kappa\left(u_{n+1}-u_{n}\right)^{2}+\frac{(1+2 \alpha)\left(1-\cos u_{n}\right)}{1+\alpha\left(1-\cos u_{n}\right)}\right] .
$$



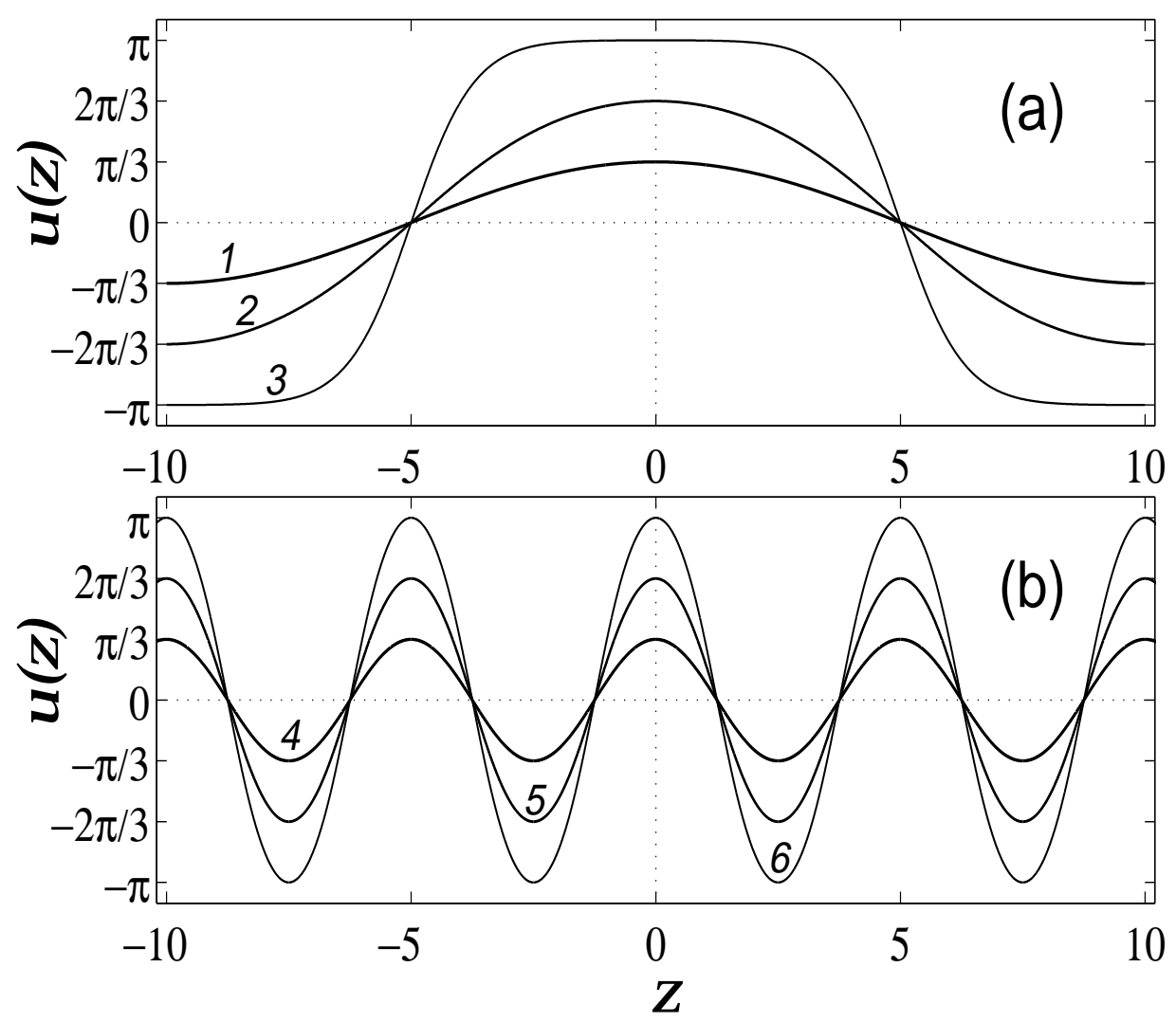

Fig. 3. Profile of the nonlinear wave with (a) $\lambda=20: A=\pi / 3$ ( $s=3.1288$, curve 1), $A=2 \pi / 3$ ( $s=2.5237$, curve 2$), A=\pi(s=0.2161$, curve 3$)$ and (b) $\lambda=5$ : $A=\pi / 3(s=1.1938$, curve 4$), A=2 \pi / 3$ ( $s=1.1029$, curve 5$), A=\pi(s=0.9911$, curve 6$)$. In both the cases $\alpha=0$ and $\gamma=1$.

In general, topological solitons (kinks and antikinks) are pinned to the lattice, so that a non-zero PN potential barrier appears. Its height measures the activation energy for the kink propagation through one lattice site. However, as shown in refs. [13,14], for sufficiently large convexity of the on-site piecewise parabolic potential, there exists a finite number of values of the coupling parameter $\kappa$ for which the kink motion becomes free. In this case the PN potential barrier completely disappears.

Let us study this phenomenon more precisely. Let $u_{n}^{K}, n \in \mathbb{Z}$, be an (anti)kink profile obtained by solving the stationary version of the Klein-Gordon equation energy (12). Then we can define the position of the kink centre

$$
\bar{n}=\sum_{n \in \mathbb{Z}} n q_{n}, \quad q_{n}=\frac{u_{n+1}^{K}-u_{n-1}^{K}}{2\left(u_{\infty}^{K}-u_{-\infty}^{K}\right)}, \quad u_{ \pm \infty}^{K}=\lim _{n \rightarrow \pm \infty} u_{n}^{K}
$$


and the kink width

$$
D=2 \sqrt{\sum_{n \in \mathbb{Z}}(n-\bar{n})^{2} q_{n}}
$$

The symmetric kink profiles are centred either exactly on a lattice site (we call them site-centred kinks or antikinks) or in the middle between two lattice sites (called bond-centred ones). The symmetry of the site-centred kink (on the site $n_{0}=\bar{n}$ ) is described by the relation

$$
u_{n_{0}-n}^{K}-\pi=\pi-u_{n_{0}+n}^{K}, \quad n \in \mathbb{Z},
$$

while for the bond-centred kink [between the $n_{0}$ th and the $\left(n_{0}+1\right)$ th sites] the symmetry relation is

$$
u_{n_{0}-n}^{K}-\pi=\pi-u_{n_{0}+n+1}^{K}, \quad n \in \mathbb{Z} .
$$

Let $E_{0}$ be the energy of a site-centred (anti)kink and $E_{1 / 2}$ be the energy of this kink, but now centred between lattice sites. In order to find the shape of the PN barrier, we have to solve the following minimisation problem:

$$
E=\sum_{n=1}^{N-1} \rightarrow E_{n} \min _{u_{1}=0, u_{N}=2 \pi}
$$

with the constraint $u_{N / 2}=\rho(0<\rho<2 \pi)$. The problem (17) has been solved by using the conjugate-gradients method, starting from the initial condition

$$
u_{n}=0, \quad \text { for } n<N / 2 ; \quad u_{N / 2}=\rho ; \quad u_{n}=2 \pi, \quad \text { for } n>N / 2 \text {. }
$$

For reasonable numerical accuracy of results obtained by this method, the number of chain sites $N$ should be 10 times greater than the average kink width $D$. Then the kink profile does not depend on the chain length. With monotonic decrease of the parameter $\rho$, the position of the kink centre $\bar{n}(\rho)$ will increase monotonically. As a result, it is possible to find the dependence of the kink energy $E$ on its position $\bar{n}: E(\bar{n})=E[\rho(\bar{n})]$.

The shape of the PN potential normalised to the minimal kink energy $E_{\min }=$ $\min E(\bar{n})$ is given in Fig. 4. As demonstrated by this figure, the PN potential is a periodic function with the period 1 . Depending on the system parameters $\kappa$ and $\alpha$, a minimum of the PN potential corresponds to the static kink centred either at a site $\left(E_{\min }=E_{0}\right)$ or between sites $\left(E_{\text {min }}=E_{1 / 2}\right)$. As can be seen from Fig. 4, by changing system parameters, namely, the coupling constant $\kappa$, one can obtain the switching of the kink ground state. Indeed, for $\kappa=0.97$ the ground state is $E_{\min }=E_{1 / 2}$ corresponding to the bond-centered kink whereas 


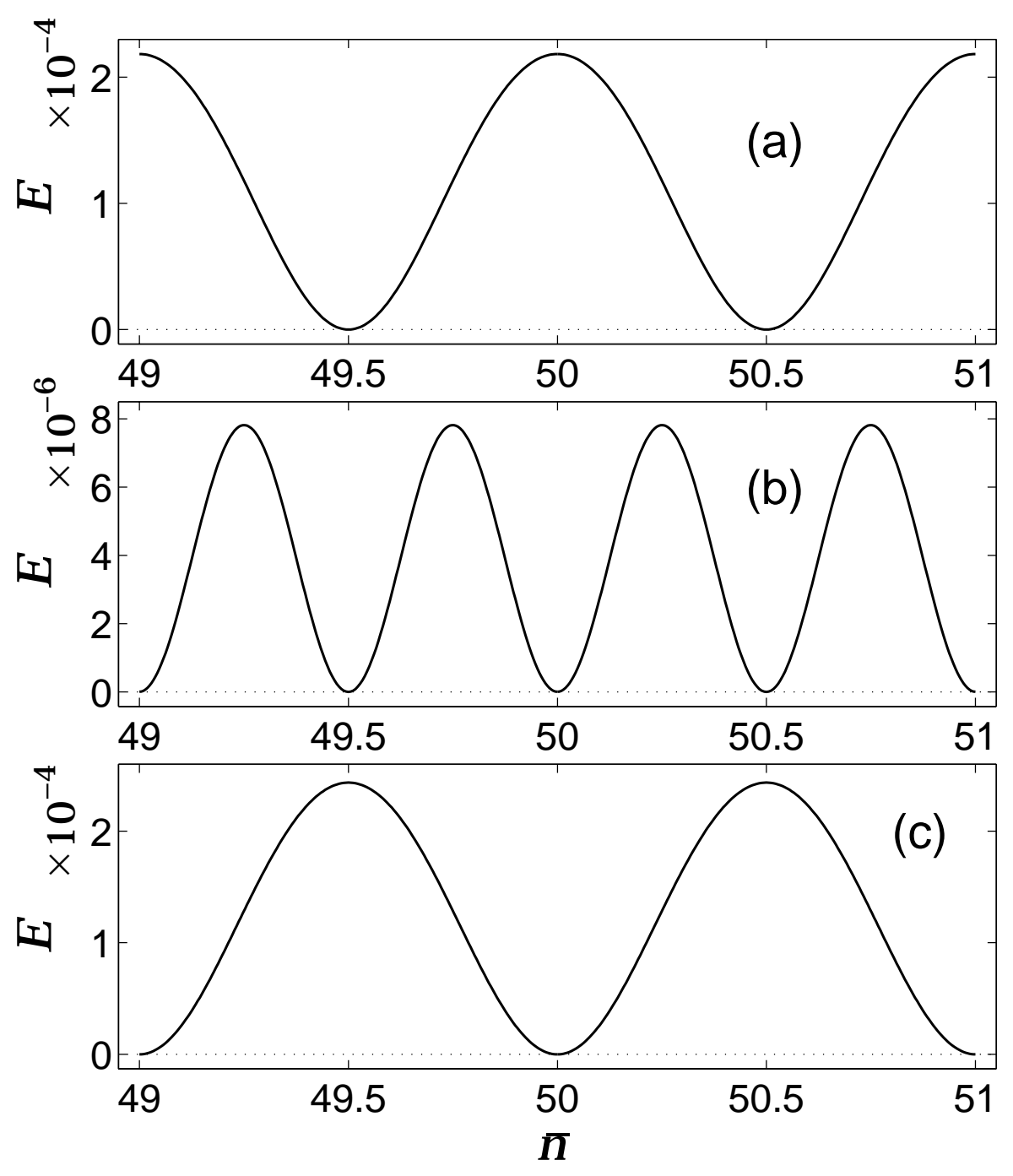

Fig. 4. Peierls-Nabarro (PN) potential relief for (a) $\kappa=0.97\left(E_{\min }=E_{1 / 2}\right)$, (b) $\kappa=0.9790104\left(E_{\min }=E_{0}=E_{1 / 2}\right)$ and $(\mathrm{c}) \kappa=0.99\left(E_{\min }=E_{0}\right)$. In all cases $\alpha=0.1$.

the site-centered kink has an energy maximum (see Fig. 4a). For $\kappa=0.979$ both the site- and bond-centred kinks are ground states $\left(E_{\min }=E_{0}=E_{1 / 2}\right)$ and the kink solution corresponding to the energy maximum lies in-between these two configurations (see Fig. 4b). Finally, in Fig. 4c for $\kappa=0.99$ we have the PN potential opposite to the case with $\kappa=0.97$.

Let us study this switching phenomenon with more details. In Fig. 5 we plot the dependence of the energy difference $\Delta E=\left|E_{0}-E_{1 / 2}\right|$ on the coupling parameter $\kappa$ for different values: $\alpha, \alpha=0.01, \alpha=0.1$. It follows from this figure that the barrier height $\Delta E$ decays exponentially with the growth of the coupling $\kappa$ for all values of the convexity parameter $\alpha$. Note that for the SG potential $(\alpha=0), \Delta E$ is always non-zero, so that for this case the bondcentred state appears to be the ground state of the chain. A small increase 

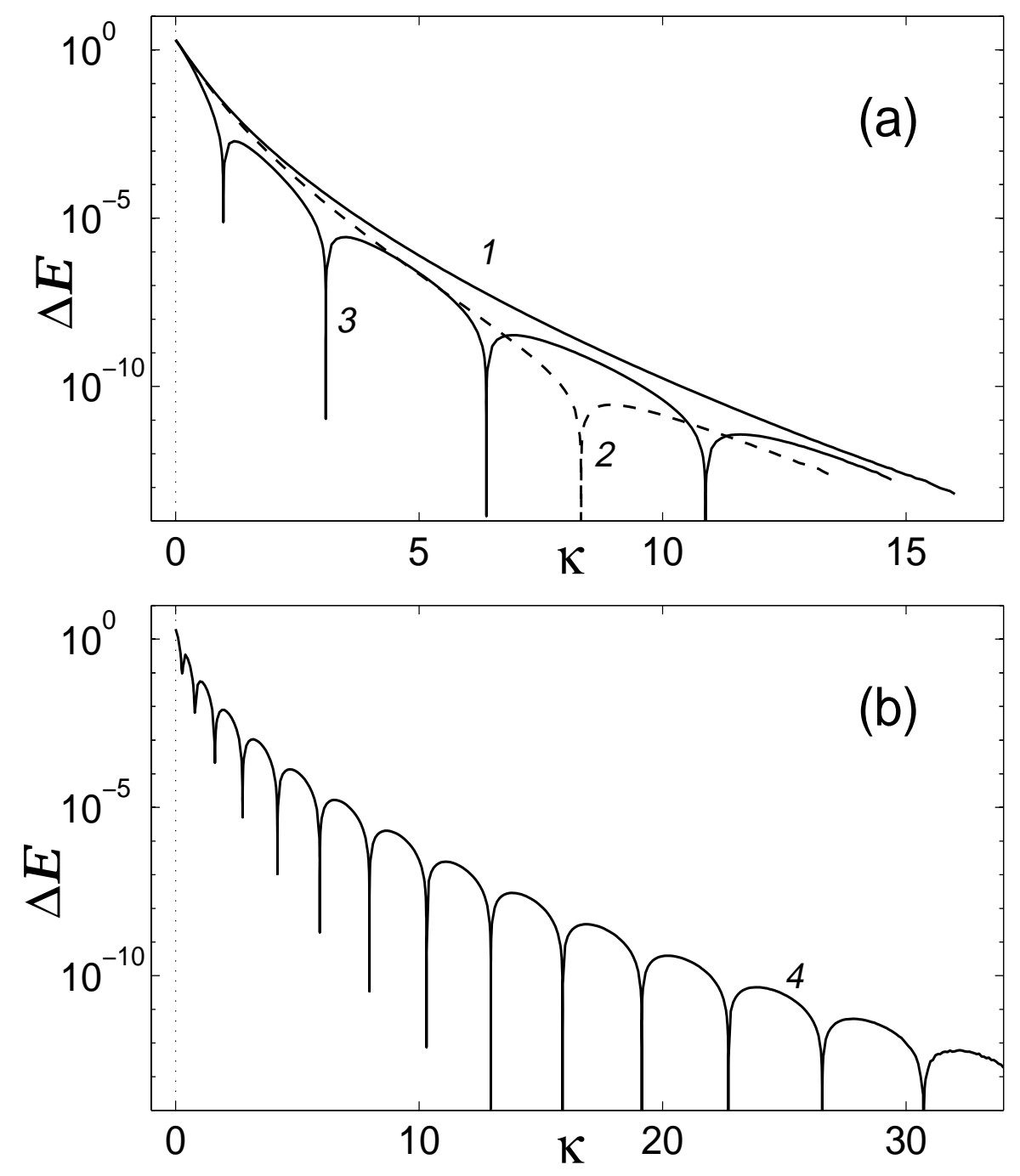

Fig. 5. Height of the barrier $\Delta E$ against the coupling $\kappa$ for the convexity parameter values: (a) $\alpha=0$ (curve 1), $\alpha=0.01$ (curve 2), $\alpha=0.1$ (curve 3) and (b) $\alpha=1$ (curve 4) .

of the convexity parameter $(\alpha=0.01)$ gives the coupling parameter value $\kappa_{1}=8.3257$ for which $E_{0}=E_{1 / 2}$. For this value of $\kappa$ the switching of the symmetry of the kink ground state occurs. The pinning energy (the hight of the PN barrier) abruptly decreases by several orders. For all $\kappa<\kappa_{1}$, the ground state of the chain is a bond-centred profile, while for $\kappa>\kappa_{1}$ the ground state has a (anti)kink profile centred on a lattice site. A further increase in $\alpha$ results in the growth of the coupling parameter values $\kappa_{l}, l=1,2, \ldots, \nu$ for which the energy (12) does not depend on the position of the (anti)kink centre. Thus, for $\alpha=0.1$, the number of states for which $E_{0}=E_{1 / 2}$ is $\nu=4$ while for $\alpha=1$ the number of such states is $\nu=14$. With the growth of $\alpha$, the points $\kappa_{l}$ shift monotonically to the left, and additional points $\kappa_{l}$ for which $E_{0}=E_{1 / 2}$ appear from the right. For each value $\kappa=\kappa_{l}$, the switching of the ground state symmetry occurs. For example, for $0 \leq \kappa<\kappa_{1}$ the ground 
state of the chain is a standing (anti)kink state with bond-centred symmetry, for $\kappa_{1}<\kappa<\kappa_{2}$ that with site-centred symmetry, for $\kappa_{2}<\kappa<\kappa_{3}$ that with bond-centred symmetry and so on. For each "transparent" point $\kappa=\kappa_{l}$, the pinning energy decreases by several orders, so that the chain becomes more "transparent" for kink motion. The dependence of the transparent points $\kappa_{l}$ on the convexity parameter $\alpha$ is given in Table 1.

Table 1

Dependence of the transparent points $\kappa_{l}, l=1,2, \ldots, \nu$ on the convexity parameter $\alpha$.

\begin{tabular}{c|ccccc}
$\kappa \backslash \alpha$ & 0 & 0.01 & 0.1 & 1 & $\infty$ \\
\hline$\kappa_{1}$ & $\infty$ & 8.3257 & 0.9790 & 0.2667 & 0.2026 \\
$\kappa_{2}$ & & $\infty$ & 3.0834 & 0.7872 & 0.6079 \\
$\kappa_{3}$ & & & 6.3808 & 1.6140 & 1.2159 \\
$\kappa_{4}$ & & & 10.881 & 2.7470 & 2.0264 \\
$\kappa_{5}$ & & & $\infty$ & 4.1819 & 3.0396 \\
$\kappa_{6}$ & & & & 5.9186 & 4.2555 \\
$\kappa_{7}$ & & & & 7.9576 & 5.6740 \\
$\kappa_{8}$ & & & & 10.2993 & 7.2951 \\
$\kappa_{9}$ & & & & 12.9440 & 9.1189 \\
$\kappa_{10}$ & & & & 15.8918 & 11.1453 \\
$\kappa_{11}$ & & & & 19.1430 & 13.3744 \\
$\kappa_{12}$ & & & & 22.1430 & 15.8061 \\
$\kappa_{13}$ & & & & 25.5500 & 18.4405 \\
$\kappa_{14}$ & & & & 30.7100 & 21.2774 \\
$\kappa_{15}$ & & & & $\infty$ & 24.3171 \\
& & & &
\end{tabular}

In order to understand the regime of free kink propagation, let us consider the limiting case when the convexity parameter $\alpha \rightarrow \infty$. In this case, for each value of $\kappa$, a countable set of kink states exists. Each state is uniquely defined by the number of particles $j$ which are located on the inter-well barrier of the on-site potential $V(u ; \infty)$. In the case of even $j$ the kink is centred between chain sites while for odd $j$ its centre is located on a chain site. The energy of the (anti)kink state with $j$ particles on the barrier is

$$
E_{j}=E_{j}(\kappa)=2 j+\frac{2 \pi^{2}}{j+1} \kappa
$$


Therefore, the symmetry switching of the ground state occurs for

$$
\kappa=\kappa_{j}=\frac{j(j+1)}{\pi^{2}}
$$

when a new chain particle appears on the potential barrier: $E_{j-1}(\kappa)=E_{j}(\kappa), j=$ $1,2, \ldots$ For instance, when $0 \leq \kappa<\kappa_{1}$, the ground state energy is $E_{0}(\kappa)$, for $\kappa_{1}<\kappa<\kappa_{2}$ it is $E_{1}(\kappa)$ and in the general case the ground state energy $E_{j}(\kappa)$ corresponds to the interval $\kappa_{j}<\kappa<\kappa_{j+1}$. Therefore, one can conclude that the transparent points $\kappa_{l} \rightarrow l(l+1) / \pi^{2}$ as the convexity parameter $\alpha$ tends to infinity.

\section{$5 \quad$ Families of moving kinks}

As we have already seen, in general it is not possible to find kinks (and antikinks) with monotonic asymptotics which propagate in a discrete lattice with permanent profile and velocity, except for some fixed values of velocity. However, their uniform propagation is possible on an oscillating background. The existence of transparent points for the coupling parameter $\alpha$ gives us reason to expect that for considerably large convexity of the substrate potential $V(u ; \alpha)$, regimes of free kink propagation can occur. We look for kink solutions of eq. (6), both with non-oscillating and oscillating asymptotics, using the pseudo-spectral method developed in $[2,20]$ and described in details in ref. [3].

\subsection{Kinks with non-oscillating asymptotics}

Let us consider the case of antikink solutions (the same results are valid for kink solutions due to the kink-antikink symmetry) with topological charge $Q=-1$. We take the fixed value: $\gamma=\kappa^{-1}=1$. For the standing profile $\Delta E=$ 0 if $\alpha=\alpha_{1}=0.0975$ and $\alpha=\alpha_{2}=0.5387$. This means that the anti(kink) should also possess a travelling-wave solution with some fixed velocities $s>0$. Therefore one can expect that for other values of $\alpha$ which are close to $\alpha_{1}$ or $\alpha_{2}$, the velocity of the anti(kink) is different from zero.

Using the pseudo-spectral method, the numerical search for (anti)kink solutions of eq. (6) with $\gamma=1$ has shown that non-oscillating (anti)kinks exist only for $\alpha \geq \alpha_{1}$. The shift of the transparent point to the right is expected because the coefficient $\gamma$ is effectively reduced, approximately by the factor $\left(1-s^{2}\right)^{-1}$ in the case of sufficiently broad (anti)kink profiles. We have found that there exists one non-zero value of velocity $s_{1}=s_{1}(\alpha)$ for which the non-oscillating 


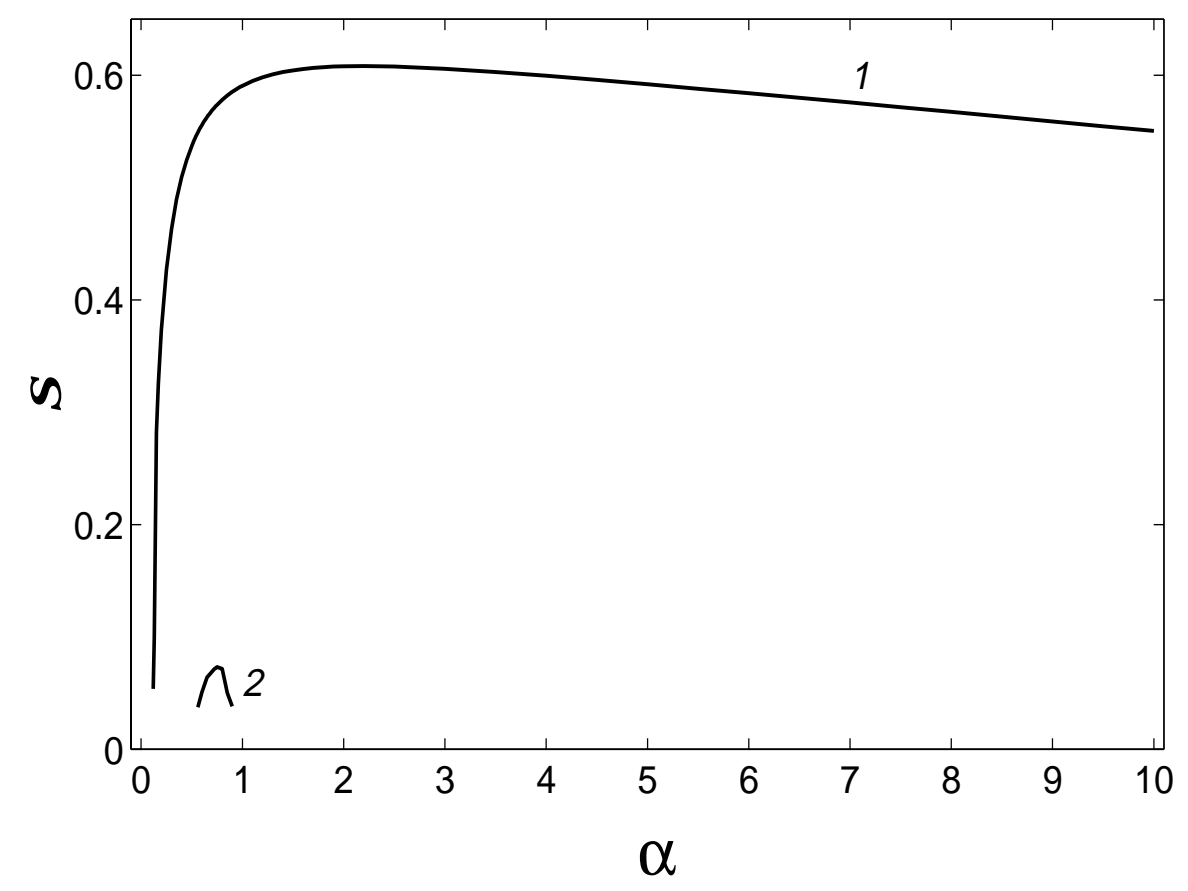

Fig. 6. Velocities $s_{1}$ (curve 1) and $s_{2}$ (curve 2) of the non-oscillating topological soliton (kink or antikink) motion against the convexity parameter $\alpha$ for $\gamma=1$.

(anti)kink propagates with permanent shape. For $\alpha_{2} \leq \alpha \leq 0.9$ there exists also another value of the velocity $s_{2}=s_{2}(\alpha)$. Note that moving kinks do not exist at infinitesimal values of $s$ and start from some small but finite value of velocity. The dependence of the velocities $s_{1}$ and $s_{2}$ of the (anti)kink on the convexity parameter $\alpha$ is shown in Fig. 6 for $\gamma=1$. The shape of the antikink solution for $\alpha=1, \gamma=1$ and $s=s_{1}=0.5907$ is shown in Fig. 7(a) by a solid line. The kink solution is the corresponding monotonically increasing function. The particle velocities $\dot{u}_{n}$ are depicted in Fig. 7(b).

Let us check the accuracy of our solution. For this purpose we integrate numerically the system of equations of motion (4) with periodic boundary conditions $u_{n \pm N} \equiv u_{n}$ and $\dot{u}_{n \pm N} \equiv \dot{u}_{n}$, where $N=200$ is the number of particles in the chain. Numerical simulations (here and in what follows the fourth-order Runge-Kutta method with the time step $\Delta t=0.01$ is used) have shown that the topological soliton can propagate along the chain with constant velocity and shape. Thus, during the time $t=6780$ the soliton passed around the chain 20 times and its initial profile (shown by circles in Fig. 7) coincided exactly with the initial one (shown by solid line in Fig. 7).

Let us study the collision of topological solitons. To this end we simulate the system of equations of motion (4) with fixed boundary conditions for the chain consisting of $N=200$ particles. In order to diminish the influence of radiation, we introduce some friction at the chain ends by adding the term $-\Gamma \dot{u}_{n}$ with $\Gamma=0.1$ to the r.h. side of the equations of motion (4) for the 

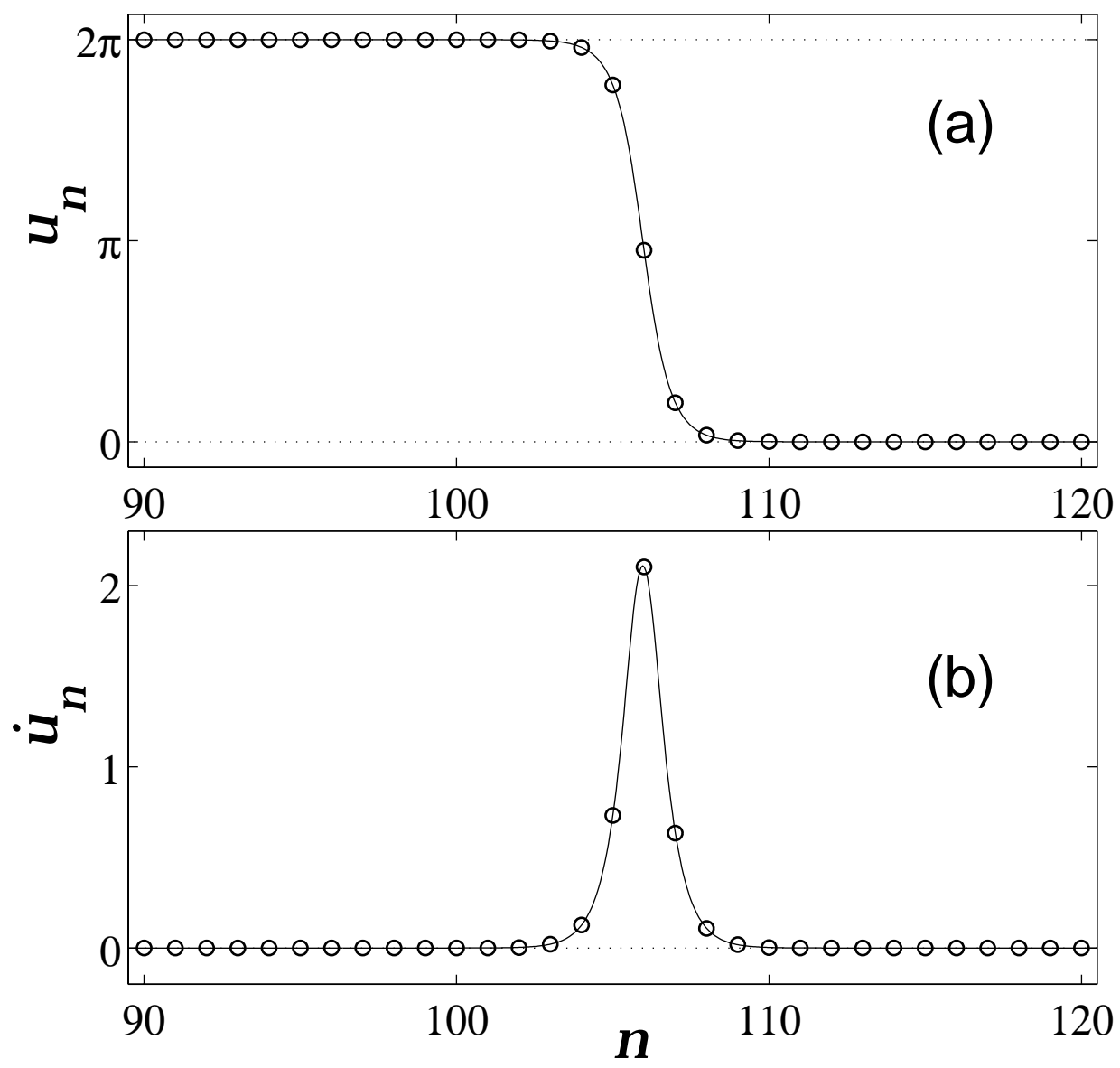

Fig. 7. Shape of the antikink for $\alpha=1, \gamma=1$ and $s=0.5907$. Solid lines represent the profiles of (a) the antikink $u(z)$ and (b) the particle-velocities field $-s u^{\prime}(z)$ obtained by using the pseudo-spectral method. Circles represent the final (a) positions $u_{n}(t)$ of the chain particles and (b) particle velocities $\dot{u}_{n}(t)$ at the time instant $t=6780$.

particles with numbers $n=1,2, \ldots, 10$ and $191,192, \ldots, 200$. We choose the initial conditions which correspond to two solitons (two antikinks) with the parameter values $\alpha=1, \gamma=1$ and $s=0.5907$ which move towards each other. The collision of the solitons with opposite topological charges results in their annihilation with the creation of a breather. However, the result of the collision of the solitons with the same topological charge is rather unexpected (see Fig. 8). This collision leads to a non-elastic reflection of the solitons which is accompanied by radiation of phonons. After the collision, the interaction of the solitons with these phonons leads to their attraction and finally to the formation of a standing bound state of these solitons that have the same topological charge (see Fig. 8 for the two colliding antikinks). This bound state consists of three components: two antikinks with a breather in-between. Therefore, in a discrete chain, stable standing bound states of topological solitons with the same charge can exist. The dynamics of such bound states will be studied below. It should be emphasised that such a scenario of the kink (or 

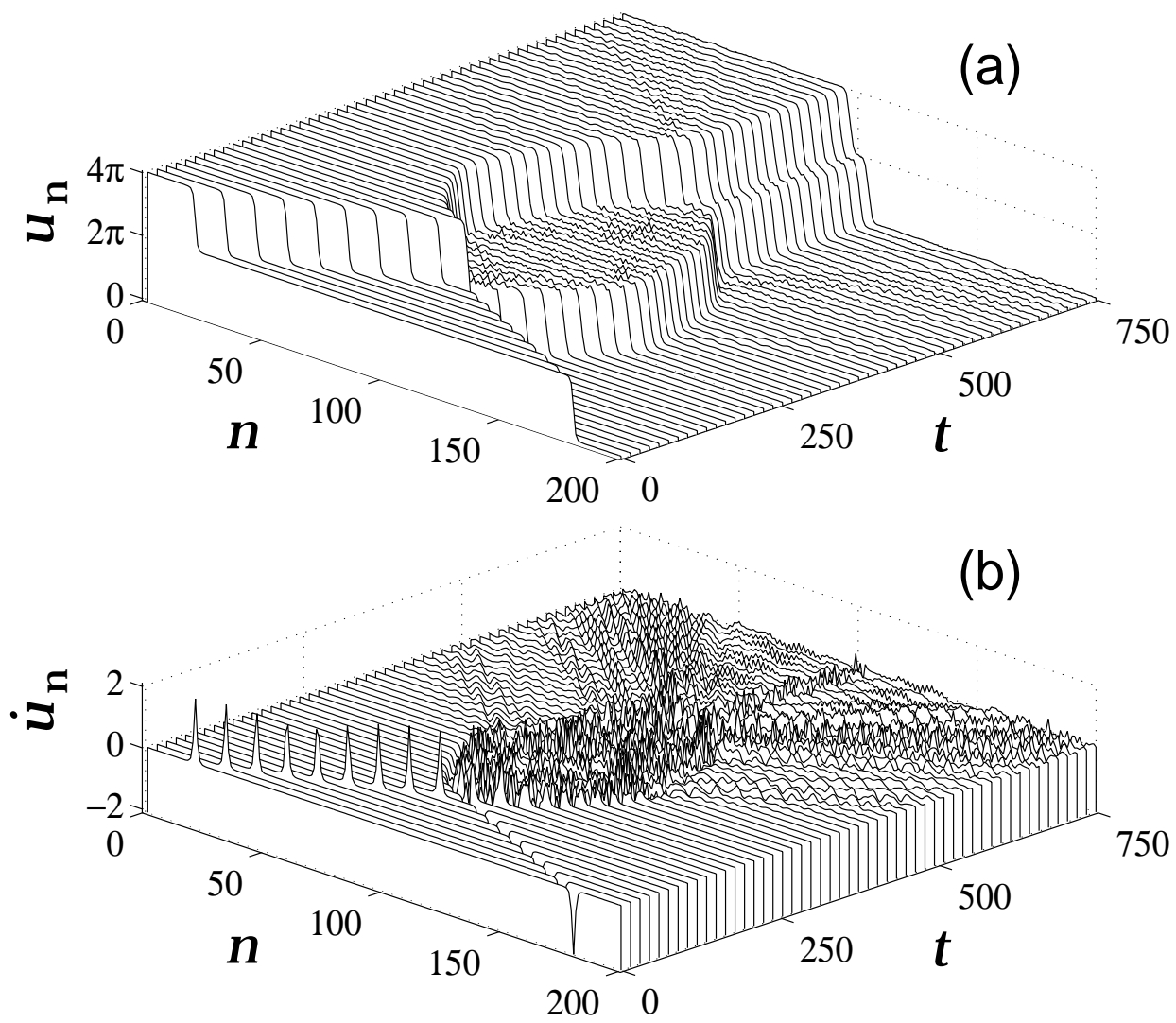

Fig. 8. Creation of the standing bound state of the two antikinks $(Q=-1)$ after their collision $(\alpha=1, \gamma=1$ and $s=0.5907)$.

antikink) collision is possible only in a discrete model. In the continuum sineGordon model such a collision always results in elastic reflection of colliding solitons.

In further stability studies we investigated collisions between kinks (or antikinks) for different values of $\alpha$. Results of these simulations show that, depending on $\alpha$, collisions can be near to elastic or non-elastic with different interaction scenarios. For small $\alpha$, collisions are close to elastic with relatively small radiation appearing as a result [see, for example, Fig. 9(a)]. For larger $\alpha$, interactions are more complicated [see Figs. 9(b) and 9(c)], with several collisions and repulsions as shown in Fig. 9(d). A similar situation was observed for the two non-integrable models: the double sine-Gordon model (see [21]) and the $\phi^{4}$ model (see [22]). Finally, for even larger $\alpha$, kinks merge together without any visible fine structure.

¿From the above results we conclude that here $\alpha$ acts in some sense as a measure of the "integrability" of a system. Indeed, when $\alpha$ is small, we are closer to the discrete sine-Gordon equation (which although still non-integrable, is close to the continuous sine-Gordon equation, which is integrable). This can be seen from mutual kink-kink (or antikink-antikink) collisions, which are elastic 

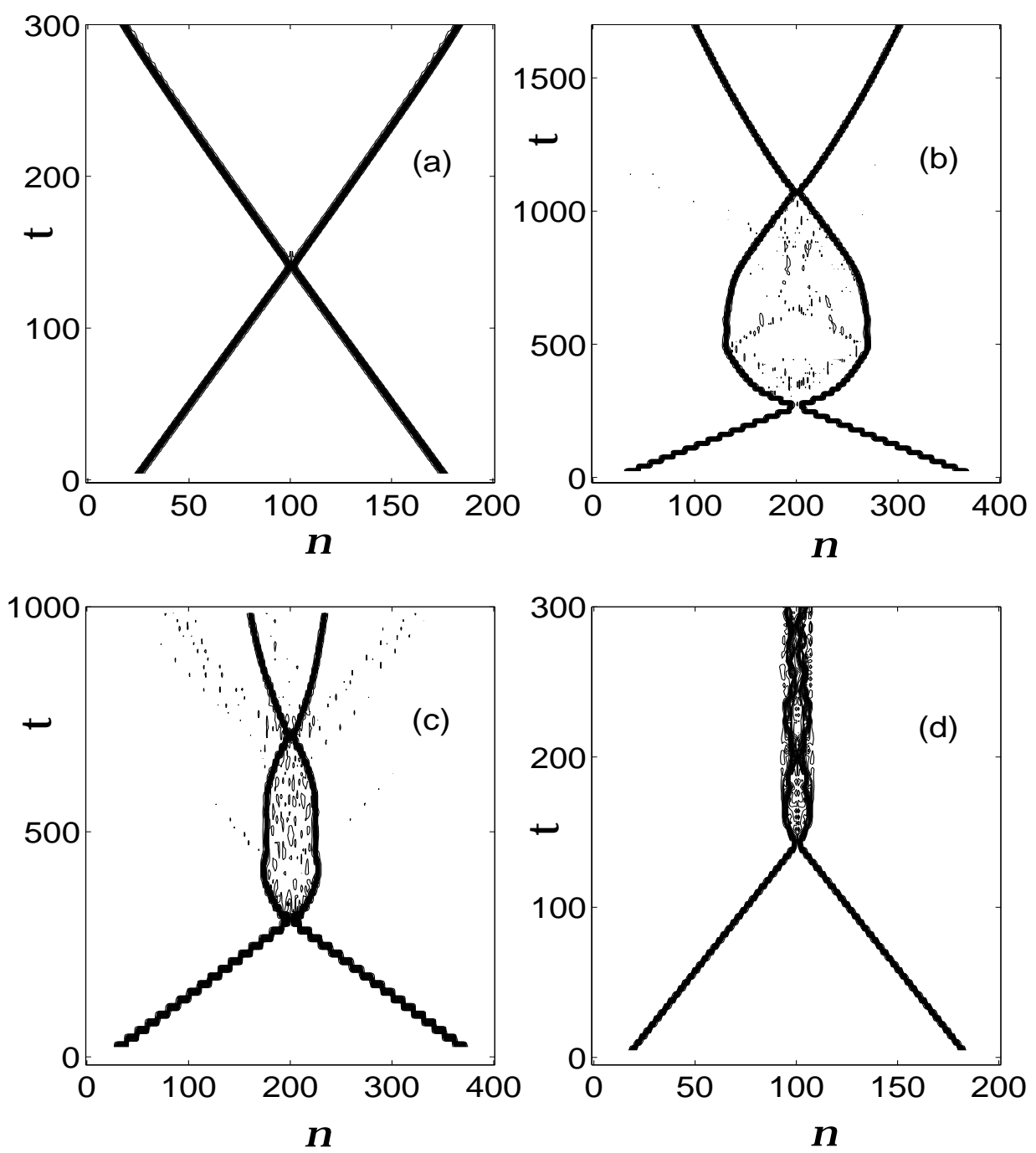

Fig. 9. Contour plots of antikink-antikink collisions for different values of $\alpha$ : (a) $\alpha=0.55, s=0.5473$; (b) $\alpha=0.75, s=0.5737$; (c) $\alpha=1.2, s=0.5982$; (d) $\alpha=5$, $=0.592$.

in integrable systems and nearly elastic for systems close to integrable. Increasing $\alpha$, we move further from the integrable model and, as a result, we obtain completely non-elastic interactions.

\subsection{Topological solitons on an oscillating background (nanopterons)}

A topological soliton (kink or antikink) with monotonic asymptotics can move along the discrete chain only with some preferred values of velocity $s$. In all other cases the soliton can move only on the background of a nonlinear periodic wave as found in ref. [2].

Let us consider the case with $\alpha=0$ and $\gamma=1$. In this case there are no 

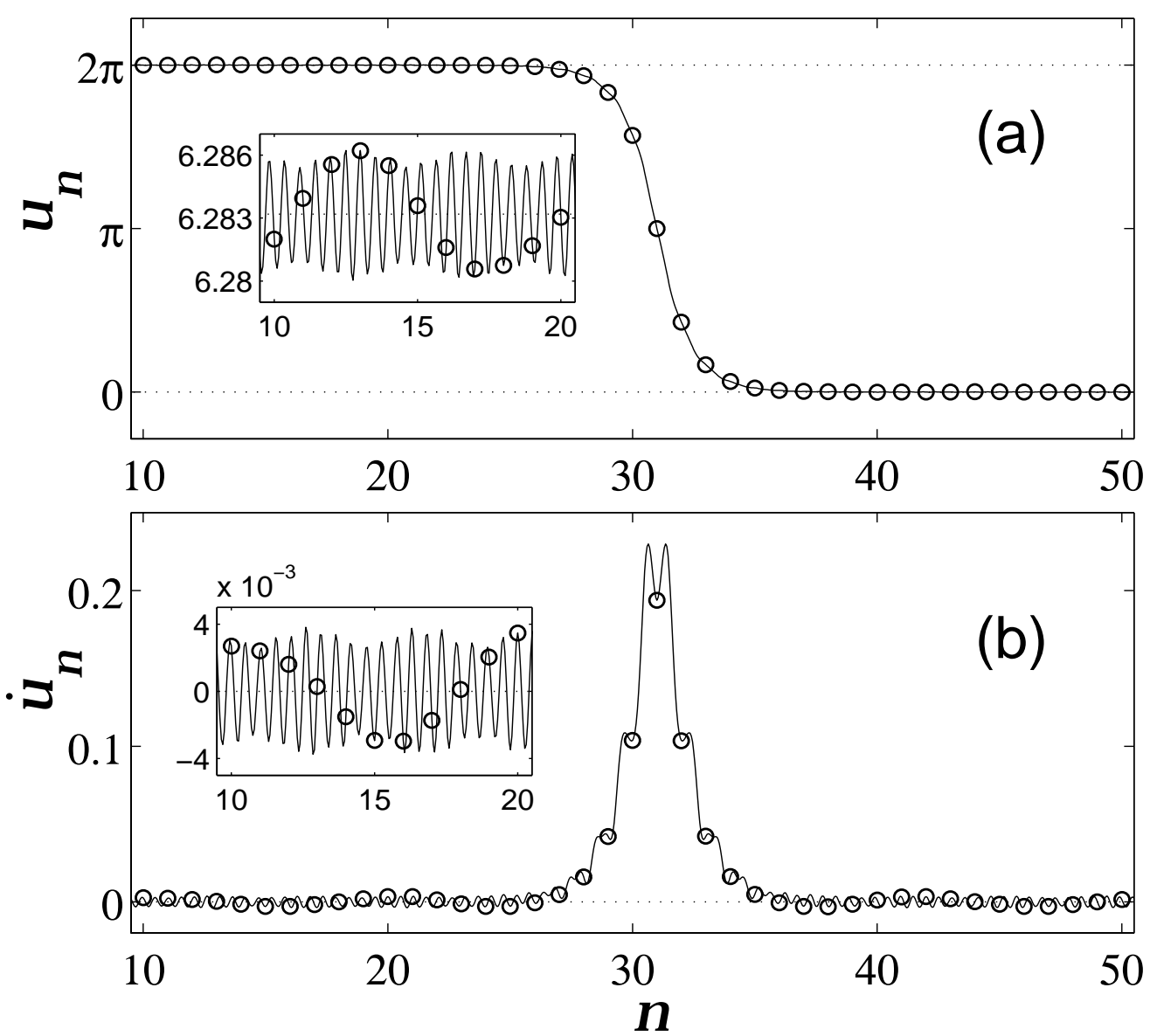

Fig. 10. Shape of the nanopteron for the parameter values $\alpha=0$ and $\gamma=1$ that moves with velocity $s=0.1$. Solid lines represent (a) the antikink profile $u(z)$ and (b) the particle-velocities field $-s u^{\prime}(z)$ obtained by using the pseudo-spectral method. Circles represent the final (a) particle positions $u_{n}(t)$ and (b) particle velocities $\dot{u}_{n}(t)$ at the time instant $t=2400$ (the chain consists of $N=60$ particles).

values of velocity $s$ when non-oscillating kink propagation is possible. The numerical search for travelling-wave solutions satisfying the condition (5) by using the pseudo-spectral method has shown that for the velocity range $0<$ $s<1$ the system of equations of motion (4) admits kink solutions on the background of a periodic wave (called nanopterons [11]). The phase velocity of these waves coincides exactly with the kink velocity. The profile of such a solution is presented in Fig. 10. As one can see from this figure, the soliton motion occurs on a background of a nonlinear modulated wave which performs de-pinning of the soliton. For $s \geq 0.72$, the wave length of the periodic wave is greater than 2 (see Fig. 2). In this case the accompanying wave becomes purely monochromatic (see Fig. 11).

It should be emphasised that the nanopteron solution is not unique for each value of velocity $s$. This non-uniqueness of the solution happens because nonlinear waves of different length can have the same velocity (see Fig. 2). The 


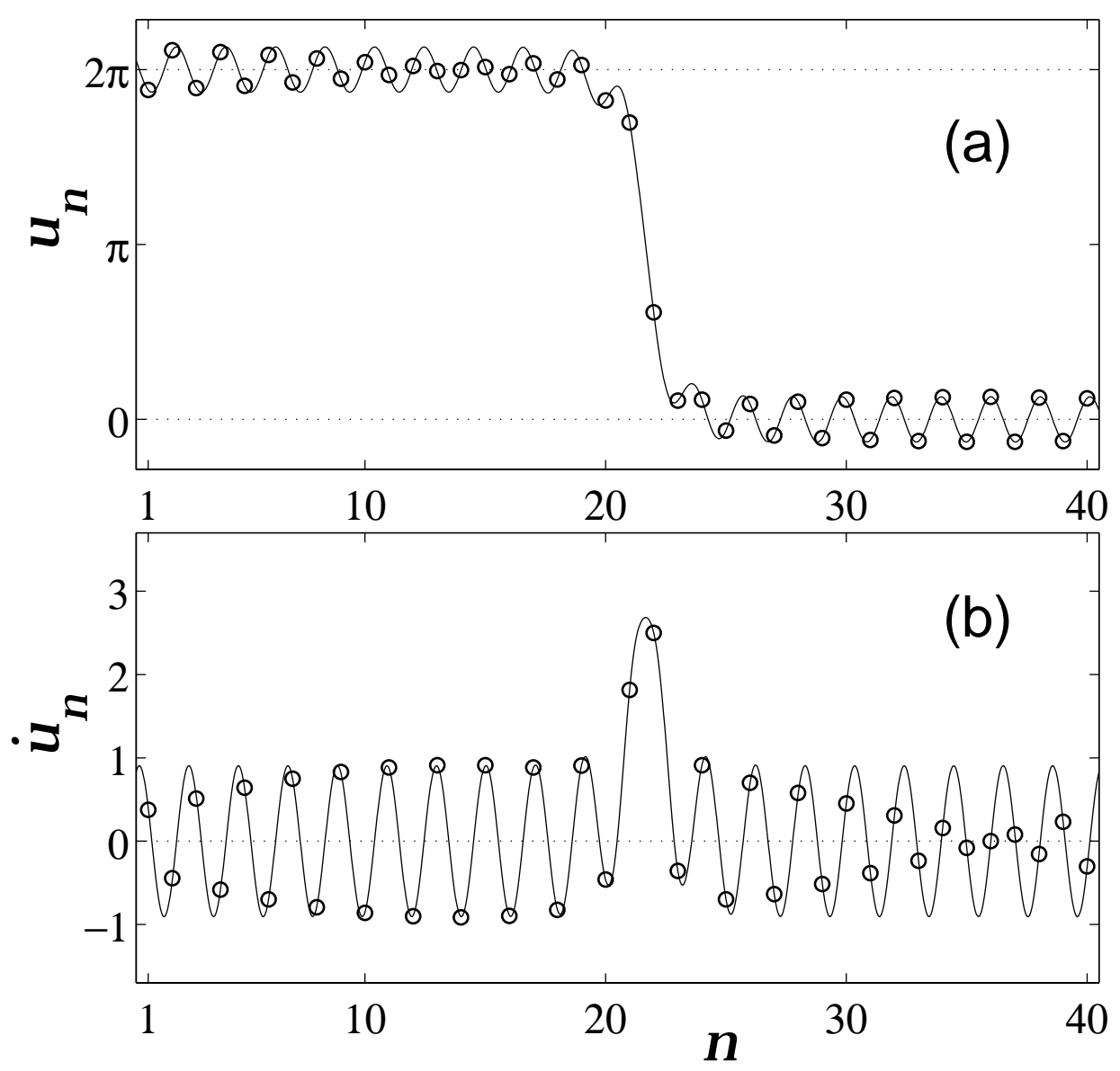

Fig. 11. Shape of the nanopteron for the system parameters $\alpha=0$ and $\gamma=1$ that propagates with velocity $s=0.73$. Solid lines represent (a) the nanopteron profile $u(z)$ and (b) the particle-velocities field $-s u^{\prime}(z)$ obtained by the pseudo-spectral method. Circles represent the final (a) particle positions $u_{n}(t)$ and (b) particle velocities $\dot{u}_{n}(t)$ at the time instant $t=1042$ (the chain consists of 40 particles). The wave length is $\lambda=2.05$ and the wave amplitude is $A=0.41$.

two different solutions for the velocity $s=0.73$ are given in Figs. 11 and 12 . The first solution corresponds to antikink motion on the background of the wave with amplitude $A=0.47$ and length $\lambda=2.05$, while the second one corresponds to the motion on the background of a wave with $A=0.557$ and $\lambda=2.053$.

The displacement and velocity fields obtained above by using the pseudospectral method were used as initial conditions for numerical simulations of the equations of motion (4) in the chain with periodic boundary conditions. These simulations have demonstrated the stability of the nanopteron profiles shown in Figs. 10 to 12 that move with velocities $s<1$. In general, the amplitude of oscillations increases with the growth of velocity $s$. Solutions of the nanopteron type also exist for $s \geq 1$. In this case the amplitude of the wave reaches $\pi$, i.e., it becomes comparable with the amplitude (height) 

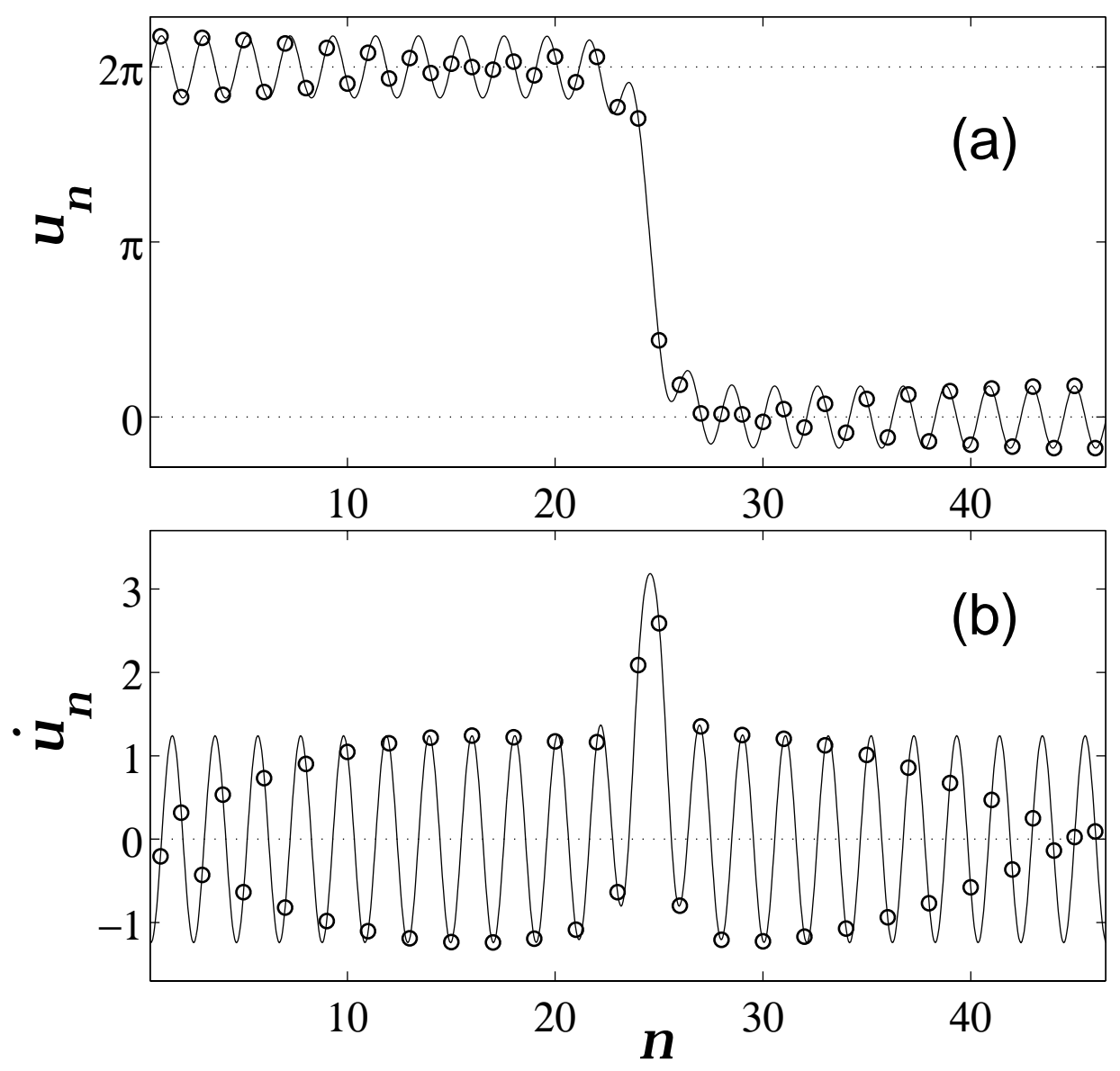

Fig. 12. Shape of the nanopteron for $\alpha=0, \gamma=1$ and $s=0.73$. Solid lines represent (a) the nanopteron profile $u(z)$ and (b) the particle-velocities field $-s u^{\prime}(z)$ obtained by using the pseudo-spectral method. Circles represent the final (a) particle positions $u_{n}(t)$ and (b) particle velocities $\dot{u}_{n}(t)$ at the time instant $t=1009$ (the chain consists of 46 particles). The wave length is $\lambda=2.053$ and the wave amplitude is $A=0.557$.

of the topological soliton. Numerical simulations of the evolution of these solutions have shown that they are unstable. During their propagation, very rapid separation of the topological soliton from the periodic wave takes place.

\subsection{Bound states of topological solitons}

Stable bound states of topological solitons of the same charge have been discovered first in ref. [1] for the discrete SG model which admits dynamically stable kink solutions with higher charges $(|Q|=2,3)$. Let us consider the case $\alpha=0$. For this case (i.e., for the standard SG model) the numerical solution of eq. (6) with the boundary conditions $u(-\infty)=4 \pi$ and $u(\infty)=0$ has shown the existence of several types of kink solutions with topological charge $Q= \pm 2$. The profile of these solutions is presented in Fig. 13. All these 

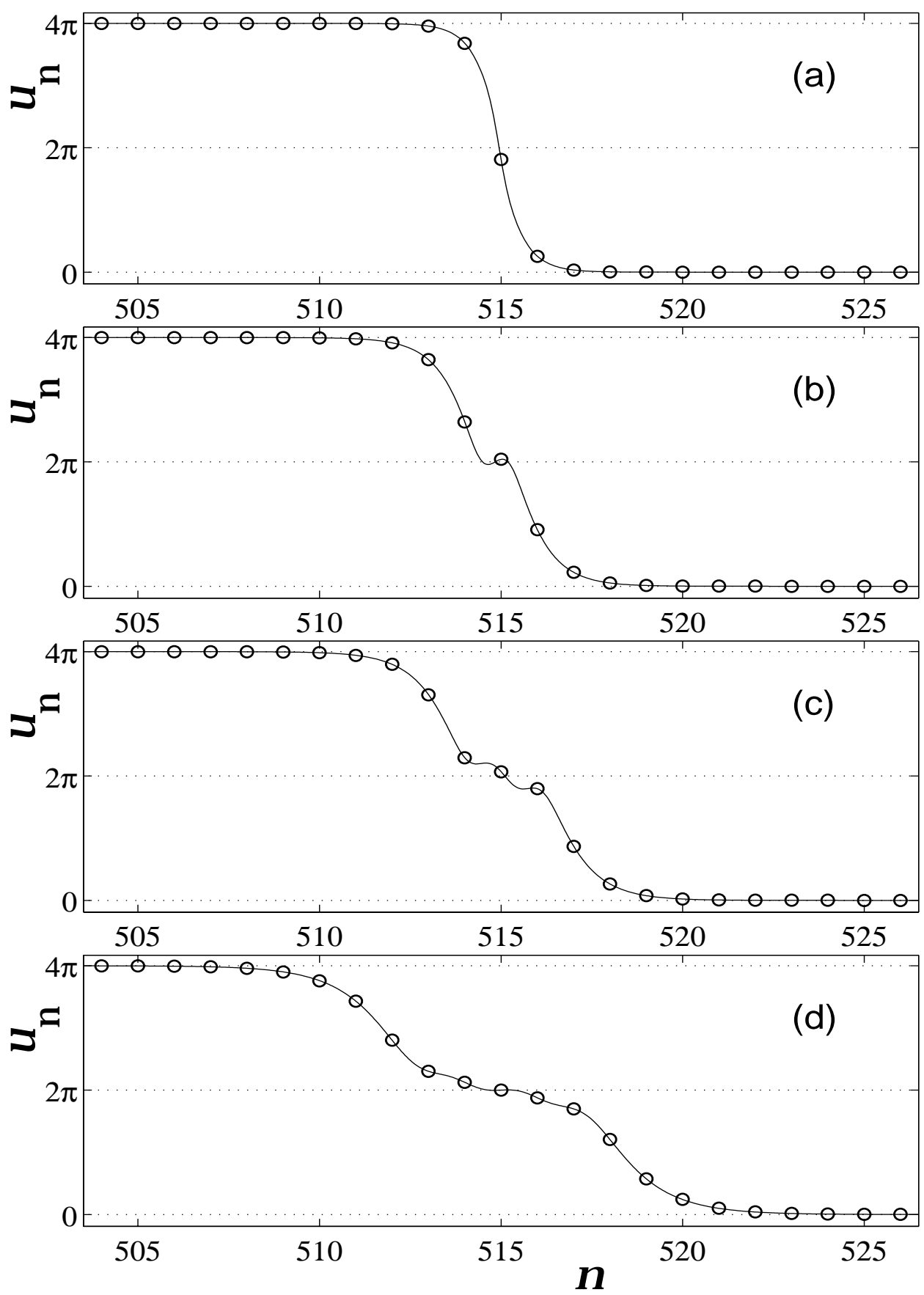

Fig. 13. Shape of the antikinks with topological charge $Q=-2$ for $\alpha=0$. Solid lines represent the soliton profile obtained by using the pseudo-spectral method. Circles represent the final particle displacements $u_{n}(t)$ obtained after integration time $t$ : (a) $k=0, \gamma=4, s=0.6134$ and $t=815.0$; (b) $k=1, \gamma=2, s=0.4724$ and $t=1058.0$; (c) $k=2, \gamma=1.5, s=0.4157$ and $t=1203.0$; (d) $k=3, \gamma=0.55$, $s=0.6155$ and $t=789.6$. 
solutions can be considered as bound states of two topological solitons and their binding can occur at different distances between them. Moreover, the profile of such a bound state in the vicinity of its centre has an odd number $2 k+1, k=0,1,2, \ldots$, of inflection points. This number (or $k$ ) determines the distance between the $2 \pi$ (anti)kinks, so that it describes the type of solutions obtained by using the pseudo-spectral method. Thus, Fig. 13(a) demonstrates the profile of the solution with zero distance between the solitons of charge $Q=-1$ ( $k=0$, one inflection point). In Fig. 13(b) the distance is longer with one lattice spacing ( $k=1$, three inflection points). The solution shown in Fig. 13(c) has a distance greater than two lattice spacings $(k=2$, five inflection points). Finally, Fig. 13(d) illustrates the solution with the inter-soliton distance greater than three lattice spacings $(k=3$, seven inflection points). The binding of the $2 \pi$ (anti)kinks is realised through the wave radiated by the first (anti)kink and absorbed by the other one. Each of these bound states has a preferred value of velocity $s_{k}, k=0,1,2, \ldots$ for each set of the system parameters. The dependence of the four velocities $s_{0}, s_{1}, s_{2}$ and $s_{3}$ on the on-site parameter $\gamma$ is illustrated by Fig. 14. The kink solution exists only for suffi-

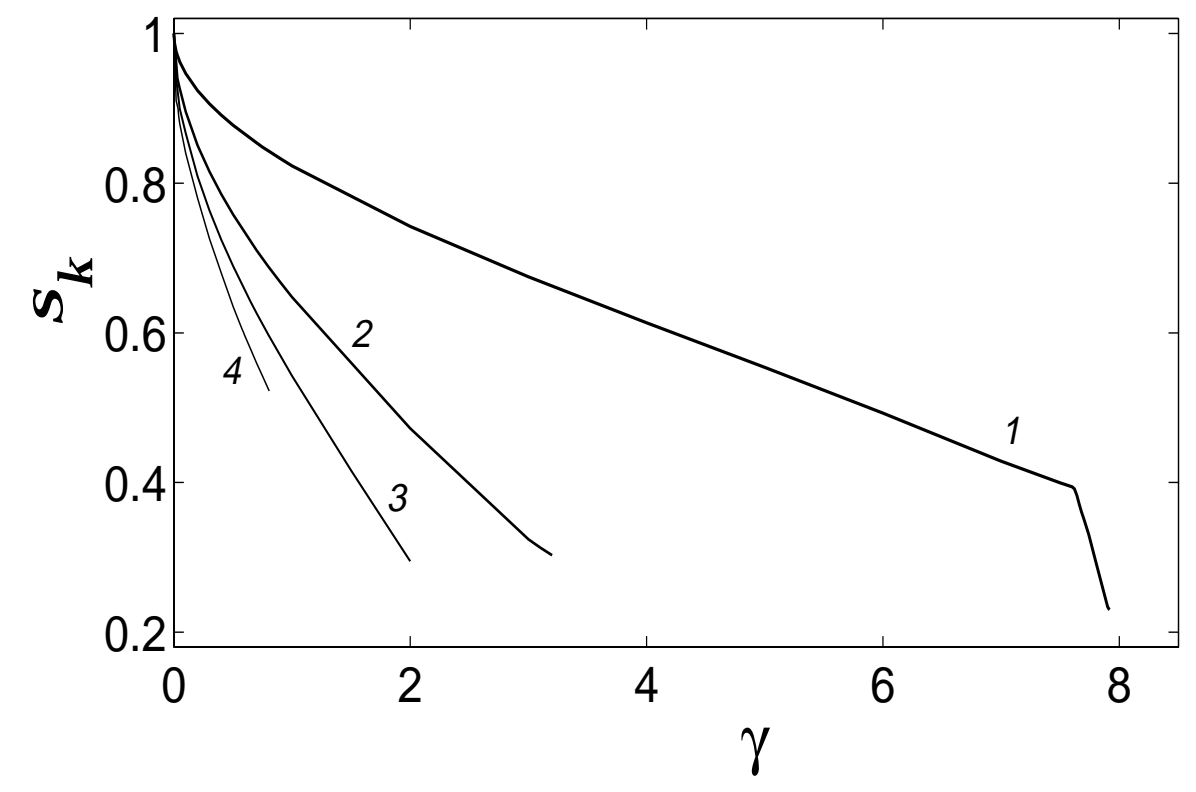

Fig. 14. Velocities $s_{k}$ for the (anti)kink with topological charge $Q= \pm 2$ against the on-site parameter $\gamma$ with $\alpha=0$ for $k=0$ (curve 1), $k=1$ (curve 2), $k=2$ (curve 3 ) and $k=3$ (curve 4 ).

ciently strong coupling parameter $\kappa$, so that $\gamma=\kappa^{-1}$ is bounded from above: $\gamma<\gamma_{k}\left(\gamma_{0}=7.9, \gamma_{1}=3.2, \gamma_{2}=2.0\right.$ and $\left.\gamma_{3}=0.8\right)$. With the growth of $\kappa$ (or the decrease of the on-site parameter $\gamma$ ), each of the preferred velocities $s_{k}$ of the (anti)kink propagation increases monotonically as shown in Fig. 14.

It should be noticed that the "transparent" velocities $s_{k}$ obtained above cannot equal zero. In other words, standing bound states of the topological solitons with $|Q|=1$ do not exist. As shown above, the existence of a localised non- 

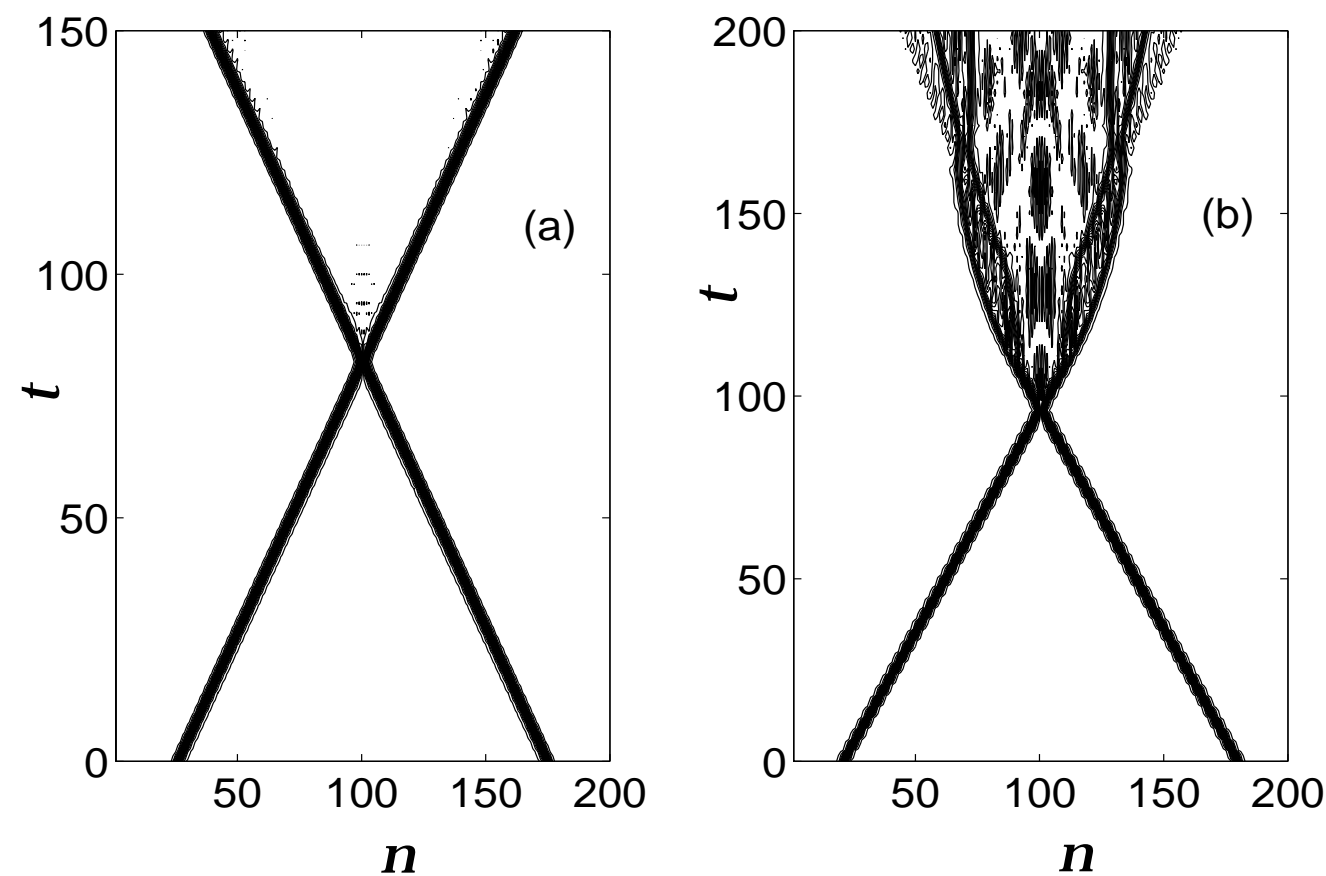

Fig. 15. Contour plots of collisions of two antikinks with topological charge $Q=-2$ for different values of $\gamma$ : (a) $\gamma=0.3, s=0.906$ and (b) $\gamma=1$ and $s=0.8233$.

linear vibration (breather) at the soliton centre is necessary for the existence of the standing bound state. Of course, the solutions of this type should also exist for $s>0$, but using the pseudo-spectral method, we are able to find only solutions of permanent profile.

The solutions with topological charge $|Q|=2$ are dynamically stable. The circles in Fig. 13 represent the particle displacements obtained by the numerical simulations of eqs. (4). The integration time was sufficiently large and, as demonstrated in Fig. 13, the final particle distributions (shown by circles) exactly coincide with the initial soliton profiles (depicted by solid lines).

We also studied the collision of the bound states of kinks (and antikinks) with $\alpha=0$. As illustrated in Fig. 15, the interaction picture depends on the parameter $\gamma$. For small $\gamma$, our system is close to the integrable sine-Gordon equation and, therefore, the collision is almost elastic [see fig. 15(a)]. For larger $\gamma$, we are further away from the continuous sine-Gordon equation and, consequently, after collision $4 \pi$ kinks (or antikinks) disintegrate into the $2 \pi$ kinks (antikinks) [see Fig. 15(b)].

The absence of elastic collisions of these bound states means that our chain is far from being an integrable system.

The same effect of the existence of fixed values of velocity $s$ was also observed for topological charges $|Q|=3$. In this case the bound state is expected to 
appear due to the three-wave resonance interaction. Some of these solutions have already been obtained in refs. [1,3]. The non-oscillating kink profiles for this case have the similar form as in the case with $|Q|=2$.

\section{Discussion and conclusions}

We have shown that the uniform propagation of narrow (anti)kinks with both monotonic and oscillating asymptotics can take place in the discrete nonlinear Klein-Gordon chain. The motion of the kinks on an oscillating background (nanopterons) happens for any given sub-sonic velocity in the Klein-Gordon lattice with arbitrary on-site potential and the nanopterons are generic solutions of such a system. From the physical point of view, this motion can be considered as a nonlinear interaction of a kink with a wave propagating along the chain. As a result of this interaction, a bound state of the kink and the wave is formed, so that the wave "pushes" the kink, on one hand, and the wave appears to be pinned to the kink, on the other hand. As a result of this kink-wave interaction, the wave velocity is reduced to sub-sonic values. In other words, some kind of "self-trapping" of a kink and a wave takes place. The numerical simulations of the equations of motion have demonstrated the stability of such a bound (anti)kink-wave state which propagates as a whole object and has a stationary profile.

The oscillating background is simply a nonlinear periodic wave which is also a generic solution of the discrete Klein-Gordon lattice. When the amplitude of this nonlinear wave is small, its shape is close to the solution of the linearised problem. Obviously, because of the nonlinear nature of such waves, their dispersion law depends on the wave amplitude. These waves resemble the cnoidal waves in continuous systems, like the sine-Gordon (SG) model.

The non-oscillating kinks appear as solutions of the nonlinear Klein-Gordon equation for some finite number of preferred values of velocity, if the corresponding time-independent problem admits discrete (anti)kink solutions when the energy difference $\Delta E$ (defined in Section 4) is zero. This happens if the convexity of the on-site potential is sufficiently large (see Fig. 1). In particular, the potential (2) for any $\alpha>0$ satisfies such a condition. The fact that moving kinks can have only discrete velocity spectrum was also confirmed recently in [23] for modified $\phi^{4}$ model where one explicit kink solution is known. Note, that in refs. [6,7] some kink-bearing systems without the PN barrier have been obtained. Kink solutions of this model can also propagate freely along the lattice. However, they were obtained in the "inverse" way by using appropriate discretisations of standard systems, like the continuous $\phi^{4}$ or SG models. As a result of such discretisations, the final systems have rather complicated forms that are not commonly used in physical applications. 
In our case we can find the values (points of transparency) of the system parameters in the realistic discrete nonlinear Klein-Gordon family of models, like the discrete SG model with a slightly modified on-site potential [see eq. (2)], at which free kink propagation in the lattice occurs. Another important point to notice is

that in contrast to the case of the nonlinear inter-particle interaction (see ref. [3]), where we were able to find non-oscillating antikinks (with supersonic or subsonic, but close to 1 , velocities), here we obtain both the kinks and antikinks with subsonic velocities and the inter-particle potential $U(r)$ in this case is harmonic. The non-oscillating antikink solutions studied in ref. [3] exist due to the inter-particle anharmonicity and therefore they have properties of both topological and acoustic (lattice) solitons.

The bound states of several simple topological solitons of charge $Q= \pm 1$ form solitons of higher charge. They also can move freely and their motion does not require any conditions on the convexity of the on-site potential. These solutions are formed as a result of the interaction of two nanopterons in such a way that their oscillating asymptotics annihilate each other. This type of the (anti)kink-(anti)kink interaction was first discovered by Peyrard and Kruskal [1]. Note that such a "bound" solution with permanent profile does not exist for zero velocity. However, in the simulations of the kink dynamics, we have obtained bound state of two standing kinks coupled through a breather. The bound states described here are not possible in the continuous Klein-Gordon model and therefore they are features of discreteness in lattice models.

\section{Acknowledgements}

One of us (Y.Z.) is grateful to Heriot-Watt University for financial support.

\section{References}

[1] M. Peyrard and M. D. Kruskal, Kink dynamics in the highly discrete sine-Gordon system, Physica D 14 (1984) 88-102.

[2] D. B. Duncan, J. C. Eilbeck, H. Feddersen and J. A. D. Wattis, Solitons on lattices, Physica D 68 (1993) 1-11.

[3] Y. Zolotaryuk, J. C. Eilbeck, and A. V. Savin, Bound states of lattice solitons and their bifurcations, Physica D 108 (1997) 81-91.

[4] S. J. Orfanidis, Discrete sine-Gordon equations, Physical Review D 18 (1978) 3823-3827.

[5] L. Pilloni and D. Levi, The inverse scattering transform for solving the discrete sine-Gordon equation, Physics Letters 92A (1982) 5-8. 
[6] J. M. Speight and R. S. Ward, Kink dynamics in a novel discrete sine-Gordon system, Nonlinearity 7 (1994) 475-484.

[7] J. Speight, A discrete $\phi^{4}$ system without a Peierls-Nabarro barrier, Nonlinearity 10 (1997) 1615-1625.

[8] A. R. Bishop, J. A. Krumhansl and S. E. Trullinger, Solitons in condensed matter: a paradigm, Physica D 1 (1980) 1-44.

[9] R. Jackiw, Quantum meaning of classical field theory, Reviews of Modern Physics 49 (1977) 681-706.

[10] G. L. Alfimov, V. M. Eleonskii, N. E. Kulagin and N. V. Mitskevich, Dynamics of topological solitons in models with nonlocal interactions, CHAOS 3 (1993) 405-414.

[11] J. P. Boyd, A numerical calculation of a weakly non-local solitary wave: the $\phi^{4}$ breather, Nonlinearity 3 (1990) 177-195.

[12] S. Flach and C. R. Willis, Discrete breathers, Physics Reports 295 (1998) 181-264.

[13] J. H. Weiner and W. T. Sanders, Peierls stress and creep of a linear chain, Physical Review A 134 (1964) 1007-1015.

[14] J. H. Weiner and A. Askar, Proton migration in hydrogen-bonded chains, Nature 226 (1970) 842-844.

[15] A. Seeger and P. Schiller, in Physical Acoustics, W. P. Mason, ed. (Academic, New York, 1966), Vol. IIIA, Chap. 8.

[16] M. Peyrard and M. Remoissenet, Soliton-like excitations in a one-dimensional atomic chain with a non-linear deformable substrate potential, Physical Review B 26 (1982) 2886-2899.

[17] R. Hobart, Peierls stress dependence on dislocation width, Journal of Applied Physics 36 (1965) 1944-1948; Peierls-Barrier Minima, Journal of Applied Physics 36 (1965) 1948-1952.

[18] A. V. Ustinov, M. Cirillo, and B. A. Malomed, Fluxon dynamics in onedimensional Josephson-junction arrays, Physical Review B 47 (1993) 8357-8360.

[19] A. V. Ustinov, B. A. Malomed, and S. Sakai, Bunched fluxon states inonedimensional Josephson-junction arrays, Physical Review E 57 (1998) 1169111697.

[20] J. C. Eilbeck and R. Flesch, Calculation of families of solitary waves on discrete lattices, Physics Letters A 149 (1990) 200-202.

[21] D. K. Campbell, M. Peyrard and P. Sodano, Kink-antikink interactions in the double sine-Gordon equation, Physica D 19 (1986) 165-205.

[22] R. K. Dodd, J. C. Eilbeck, J. D. Gibbon and H. C. Morris, Solitons and Nonlinear Wave Equations (Academic, London, 1982). 
[23] S. Flach, Y. Zolotaryuk, and K. Kladko, Moving lattice kinks and pulses: An inverse method, Physical Review E 59 (1999) 6105-6115. 